\title{
High-energy sub-nanosecond optical pulse generation with a semiconductor laser diode for pulsed TOF laser ranging utilizing the single photon detection approach
}

\author{
Jaakko Huikari $^{1}$ Eugene Avrutin ${ }^{2} \cdot$ Boris Ryvkin $^{1,3} \cdot$ Juha Kostamovaara $^{1}$
}

Received: 12 November 2015/Accepted: 22 January 2016/Published online: 13 February 2016

(c) The Author(s) 2016. This article is published with open access at Springerlink.com

\begin{abstract}
Bulk and quantum well laser diodes with a large equivalent spot size of $d_{d} / \Gamma_{a} \approx 3 \mu \mathrm{m}$ and stripe width/cavity length of $30 \mu \mathrm{m} / 3 \mathrm{~mm}$ were realized and tested. They achieved a pulse energy and pulse length of the order of $\sim 1 \mathrm{~nJ}$ and $\sim 100 \mathrm{ps}$, respectively, with a peak pulse current of $6-8 \mathrm{~A}$ and a current pulse width of $1 \mathrm{~ns}$. The 2D characteristics of the optical output power versus wavelength and time were also analyzed with a monochromator/streak camera set-up. The far-field characteristics were studied with respect to the timehomogeneity and energy distribution. The feasibility of a laser diode with a large equivalent spot size in single photon detection based laser ranging was demonstrated to a non-cooperative target at a distance of a few tens of meters.
\end{abstract}

Keywords Semiconductor laser diode - Gain switching · Laser radar - Single photon detection · Laser ranging .

Solid-state scanner

\section{Introduction}

Recently, it has been shown that the single photon detection (single photon avalanche detector, SPAD) mode presents a very interesting option for the receiver of a pulsed

Jaakko Huikari

jmth@ee.oulu.fi

1 Department of Electrical Engineering, Electronics Laboratory, University of Oulu, Linnanmaa, 90014 Oulu, Finland

2 Department of Electronics, University of York, York YO10 5DD, UK

3 A.F. Ioffe Physico-Technical Institute, 194021 St. Petersburg, Russia time-of-flight (TOF) laser radar [1-4]. The main advantages of the single photon detection compared to linear detection (e.g. based on avalanche photo detection, APD) are the sensitivity and the possibility of realizing the SPAD or even an array of SPADs on a standard CMOS integrated circuit (IC) technology. The sensitivity is strongly wavelength dependent and limited by the photon detection probability (PDP). The PDP is typically $5-2 \%$ in the wavelength range of $800-900 \mathrm{~nm}$ meaning that approximately 50 photons per pulse are needed for a valid detection. This is about an order of magnitude less than what is needed in a typical linear receiver [4]. In addition, no separate analogue amplifier channel is needed which simplifies the receiver considerably. A SPAD is essentially a reverse biased $p$ - $n$ junction with a bias voltage higher than the breakdown voltage of the junction. The detected photon introduces a fast voltage change over the junction with a magnitude of approximately $V_{\text {bias }}-V_{\mathrm{br}}$. Thus, a digital level signal is immediately achieved with a proper bias. The noise of the detector is being produced by the random detections of thermally generated charge-carriers within the depleted region of the diode, and also by the photons due to the background radiation (from the Sun) falling into the field of view of the receiver. It is important also to note that the timing jitter of a CMOS based SPAD is quite low, typically only of about 50-100 ps (FWHM) [5, 6].

The possibility of realizing a sensitive and low jitter SPAD detector or a SPAD array in a standard CMOS technology does not only simplify the radar construction, but makes it possible to suggest completely new laser radar configurations. For example, realizing a miniaturized solid-state scanner based on the pulsed time-of-flight approach and the appealing concept of focal plane scanning becomes possible. From the miniaturization point of view, this approach would benefit from the availability of semiconductor laser source producing 
highly energetic and short pulses with a characteristic pulse length on the scale of the jitter of the SPAD ( $\sim 100 \mathrm{ps})$. Radiometric calculations show that a pulse energy of $\sim 1 \mathrm{~nJ}$ is needed for non-cooperative targets within a range of tens of meters from the emitter/receiver, with the size of optics of $\sim 5 \mathrm{~cm}^{2}$ [4].

The achievable single shot precision of such a laser radar working in the single photon detection mode is set by the optical pulse width and the single photon detector response jitter. With a pulse width of $100 \mathrm{ps}$, a single shot distance measurement precision of the order of a few centimeters is available (67 ps is equivalent to $1 \mathrm{~cm}$ ). Thus, such a configuration brings forth a prospect of constructing a very compact, high speed and accurate TOFlaser radar and possibly also solid-state $2 \mathrm{D}$ and $3 \mathrm{D}$ imagers.

In the earlier work, it was shown that highly intensive and fast optical pulses can be produced with semiconductor lasers utilizing a large equivalent spot size, or the ratio $\left(d_{d}\right)$ $\Gamma_{a}$ ) of the active layer thickness $d_{a}$ to the confinement factor $\Gamma_{a}$ [7-10]. Rate equation based simulations showed that the use of a large equivalent spot size of $>2 \mu \mathrm{m}$ in the laser diode construction results in enhanced gain switching and single (after pulse-free) optical pulses with an energy in the range of a few nanojoules and a duration around 100 ps as is shown in details in the later sections of this paper. Moreover, a driving pulse with a peak current of $<10 \mathrm{~A}$ and a pulse width of $\sim 1$ ns only is needed; such pulse parameters are readily available with MOS-switch based pulsing electronics [12]. As a result, not only the SPAD based receiver, but also the semiconductor laser diode based transmitter can be miniaturized, which is highly desirable in the anticipated 3-D measuring applications.

The goal of this paper is to present and compare measured results from bulk and quantum well laser diodes utilizing the "enhanced gain switching" principle and aiming at the above mentioned performance parameters. The comparison is carried out with regard to the parameters, e.g. the temperature characteristics, which are relevant especially for the laser radar system level design. Section 2 discusses briefly the laser designs studied, and the measured results are given in Sect. 3. Some system level results are given in Sect. 4, and finally conclusions are made in Sect. 5 .

\section{Laser structures}

All lasers used in this work were of the asymmetric waveguide type investigated in our earlier work [7, 8, 10] with the refractive index contrast between the optical confinement layer (OCL) and the $p$-cladding substantially stronger than that between the OCL and the $n$-cladding, and the active layer position shifted strongly from the centre of the OCL towards the $p$-cladding. With most of the transverse mode power localized within the OCL, the lasers were of the broad asymmetric as opposed to Narrow Asymmetric waveguide type [13]. As shown previously [7, $8,13]$, lasers of this type combine the large effective spot size crucial for enhanced gain-switching, built-in single transverse mode operation, low internal losses maintained at high currents, good injection efficiency, and good fabrication tolerance. Two types of laser structures were investigated, with either quantum well (QW) or bulk active layers. The QW lasers were very similar to those used in [11]. They had an active layer in the form of five thin (4 nm thick each) GaAs/ Al $x \mathrm{Ga} 1-x \mathrm{As}(x \approx 0.3)$ QWs corresponding to a total $d a=20 \mathrm{~nm}$; the operating wavelength of these lasers was $808 \mathrm{~nm}$. The value $d a /$ $\Gamma a \approx 3 \mu \mathrm{m}$ was achieved by shifting the active layer towards the $p$-cladding beyond the position of peak modal intensity. The injection efficiency of the QW lasers was measured as $\approx 0.75$, and the internal loss, as $\approx 1.5 \mathrm{~cm}^{-1}$. The bulk lasers had a waveguide structure similar to that of QW lasers and the same $d_{a} / \Gamma_{a} \approx 3 \mu \mathrm{m}$, but with a broader waveguide layer and a GaAs active layer $80 \mathrm{~nm}$ thick; the operating wavelength was $\approx 870 \mathrm{~nm}$.

\section{Experimental}

The time domain measurements were performed to determine the relation between the drive current pulse and the optical output pulse for the bulk and quantum well semiconductor laser designs, and specifically to determine their relative positions in the time domain.

The laser diodes' drive current pulse was determined by measuring the voltage drop across a damping resistor in series with the laser diode. Optical pulse power was determined by combining the results of laser pulse time domain measurements and laser pulse train average power measurements. In the time domain measurement, the optical energy was collected with a lens pair and guided, via graded index optical fiber, to the input of a $24 \mathrm{GHz} \mathrm{OE}$ converter, whereas pulse train average power was measured with a calibrated photodiode detector.

Shown in Figs. 1 and 2 are the time domain measurement results of bulk and quantum well laser designs, respectively. Figures display separate time domain measurement results, note the drive current amplitude increasing from right to left, on the same frame for a convenient assessment. Results show driving current pulses with a amplitudes of an ampere scale and a duration of the order of $1 \mathrm{~ns}$ producing high speed, $100 \mathrm{ps,} \mathrm{laser} \mathrm{pulses,}$ with the optical power reaching up to $10 \mathrm{~W}$ with a bulk laser diode, and slightly less with a quantum well LD. 


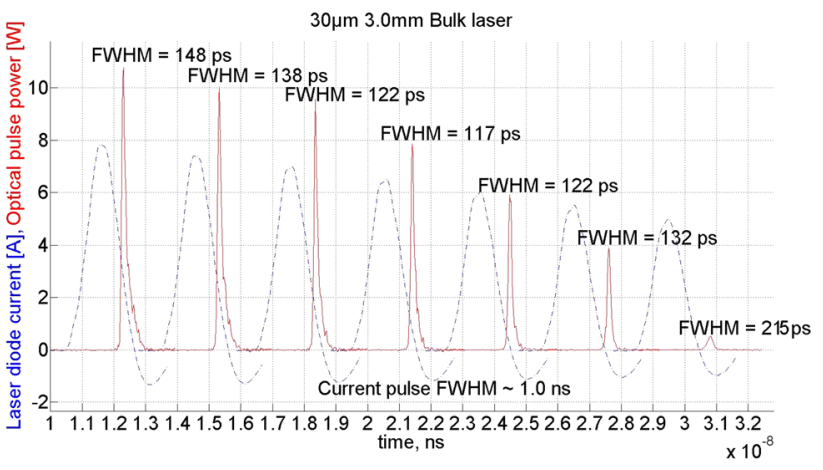

Fig. 1 Time domain measurement results of a $30 \mu \mathrm{m} 3.0 \mathrm{~mm}$ (stripe width and cavity length) bulk laser

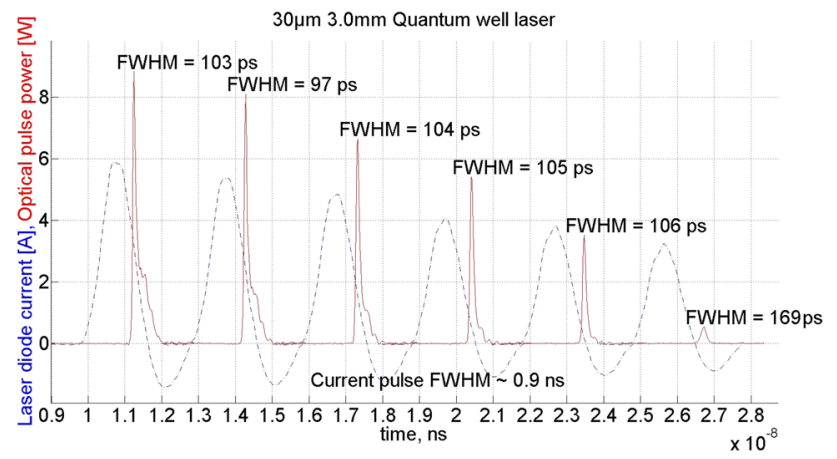

Fig. 2 Time domain measurement results of a $30 \mu \mathrm{m} 3.0 \mathrm{~mm}$ (stripe width and cavity length) quantum well laser

To determine and compare the temperature dependence of the output from the $30 \mu \mathrm{m}$ wide, $3.0 \mathrm{~mm}$ long bulk and quantum well lasers, the time domain measurements were carried out at three different operation temperatures. The temperature performance result plots (Figs. 3, 4) show the laser pulse peak value as a function of the drive current pulse peak value at the temperatures of 0,30 and $60{ }^{\circ} \mathrm{C}$. During the measurements, the laser transmitter temperature was controlled using a thermal oven. The transmitter heating up due to laser drive current pulsing is assumed to be negligible at the relatively low $10 \mathrm{kHz}$ pulsing frequency used in the measurements.

The bulk laser diode's measurement result in Fig. 3 and that of a quantum well laser diode in Fig. 4 show the achievable optical peak power being similar for both laser diode designs. However, the quantum well laser's threshold current is significantly lower (mainly because of the larger thickness of the bulk active layer). The figures also show the temperature increase causing more dramatic effect in optical output power the closer the laser's driving current peak value is to the lasing current threshold value. For instance, in the case of the bulk laser, at the drive current peak value of $6 \mathrm{~A}, 60{ }^{\circ} \mathrm{C}$ degrees temperature increase almost switches off the laser, while in the case of the

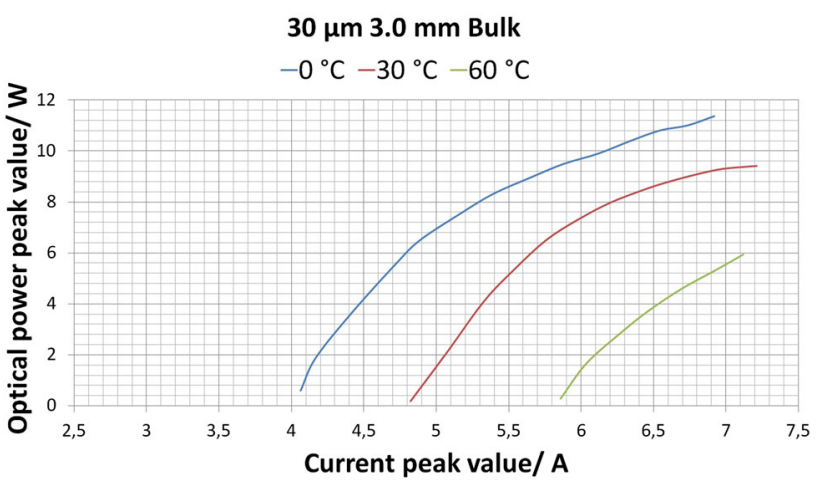

Fig. 3 The optical power peak values vs. drive current peak values of the $30 \mu \mathrm{m} 3.0 \mathrm{~mm}$ bulk laser in three different temperatures

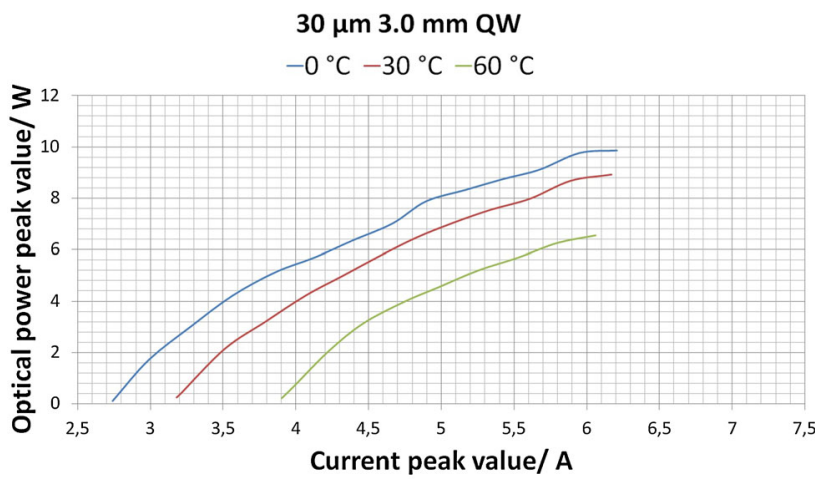

Fig. 4 The optical power peak values vs. drive current peak values of the $30 \mu \mathrm{m} 3.0 \mathrm{~mm}$ quantum well laser in three different temperatures

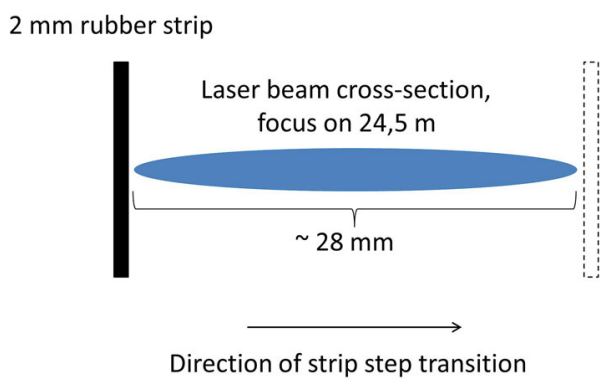

Fig. 5 The strip target spatial sweep over the laser beam at $24.5 \mathrm{~m}$

quantum well laser, the optical output power peak value decreases by $\sim 35 \%$ only. Preliminary calculations show however that the main reason for this is likely to be simply the much smaller total active layer thickness $d_{a}$ of the quantum well laser compared to that of the bulk lasers, rather than the properties of the QW material directly caused by size quantization. This means that a bulk laser with a substantially decreased $d_{a}$ may potentially offer temperature stability comparable to that of the QW lasers with the same $d_{d} / \Gamma_{a}$. 
Fig. 6 Time of flight histogram representation of spatial and time-domain energy distribution of the $30 \mu \mathrm{m} 3.0 \mathrm{~mm}$ bulk laser

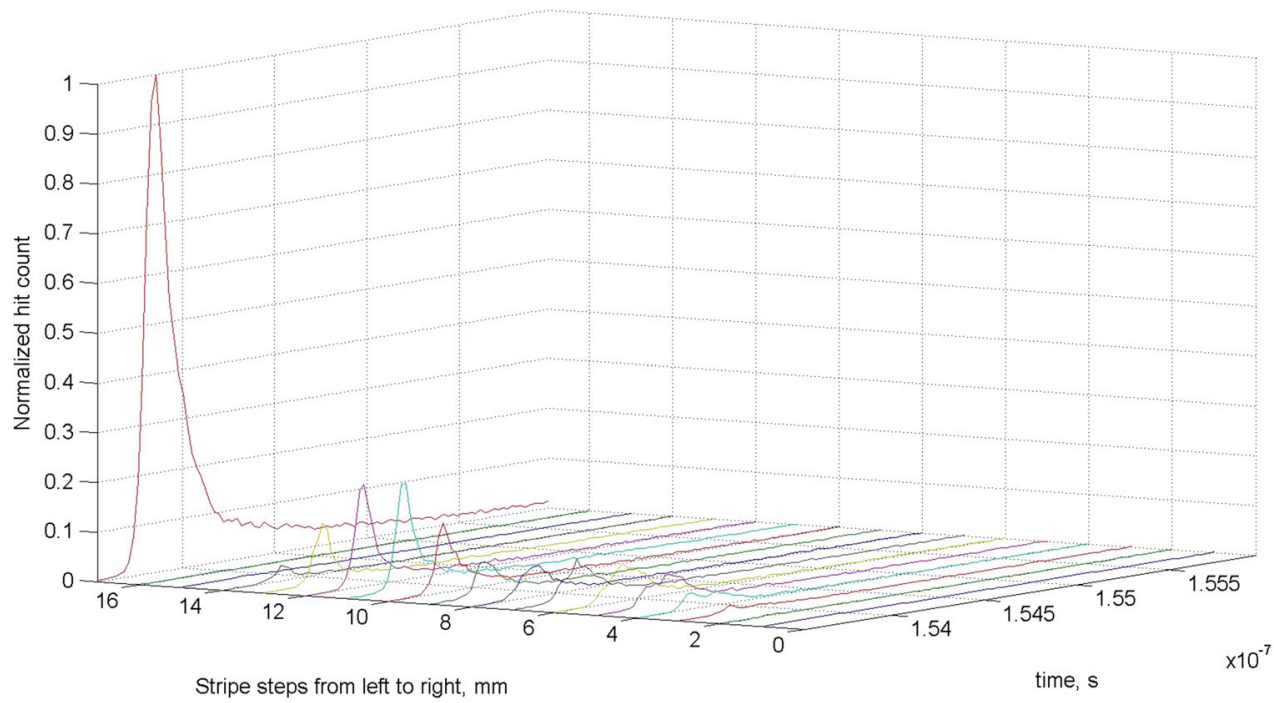

Fig. 7 Time of flight histogram representation of spatial and time-domain energy distribution of the $30 \mu \mathrm{m} 3.0 \mathrm{~mm}$ quantum well laser

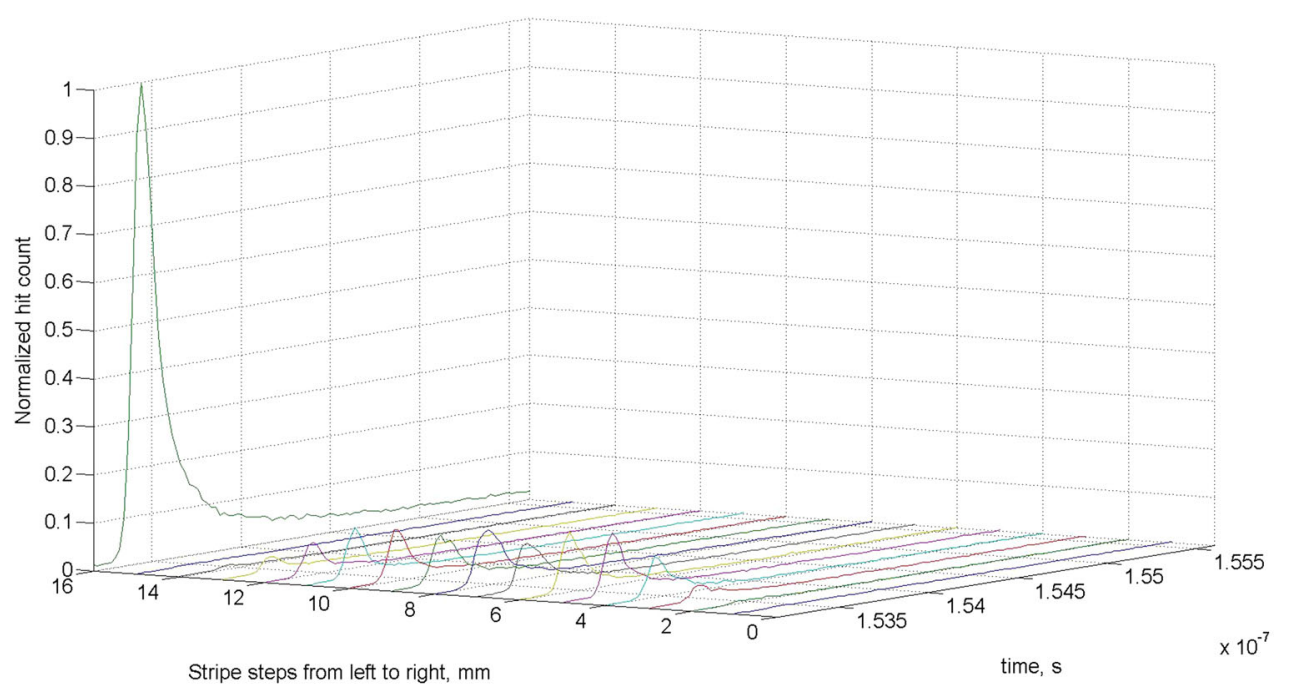

The laser beam spatial sweep measurements were performed to define and compare the spatial distribution (in the slow axis direction/plane) of the laser pulse energy, and also to measure the spatial homogeneity of the time domain behavior of emission. The measurement was done using a laboratory laser radar system, the target being a $2 \mathrm{~mm}$ wide black rubber strip (reflectance $\sim 3 \%$ ) which was swept across the focused laser beam with a width of approximately $28 \mathrm{~mm}$, at a distance of $24.5 \mathrm{~m}$ from the laser radar system. The horizontal transition step of the strip between successive measuring points was also $2 \mathrm{~mm}$. An illustration of the strip target spatial sweep setup is shown in the Fig. 5. At every measurement point, a measuring time of $30 \mathrm{~s}$ was used to accumulate a histogram of laser pulse flight times with a time interval measurement unit.

The measured detection rate of the laser radar system at both laser designs was $\sim 1 \%$ indicating that the detector operating mode was in the single photon mode. At a detection rate higher than $\sim 10 \%$, signal distortion begins to occur due to photon pile-up, since detection is a result of several photons incident on the detector [14]. Comparing the results in Figs. 6 and 7, we note that the quantum well laser diode shows more evenly distributed spatial beam energy than the bulk laser. The bulk laser diode distribution shows two energy peaks, the narrower of which one has a higher energy. Noticeable variation in the time-domain shapes of the measured hit distributions is also visible in both of the laser diode designs.

The temperature behavior of the $30 \mu \mathrm{m}$ wide, $3.0 \mathrm{~mm}$ long quantum well laser diode is thus more appealing from the practical application point of view, and thus it was selected for further studies. These included the 2-D (wavelength versus time) studies of the optical output with a monochromator/streak set-up. Also, a more accurate 


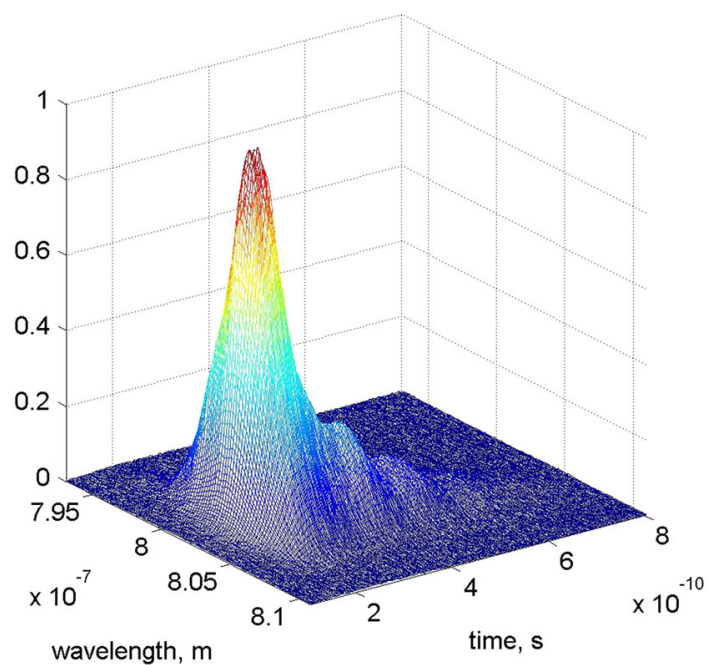

Fig. 8 The $30 \mu \mathrm{m} 3.0 \mathrm{~mm}$ quantum well laser diode's optical output power as a function of time and wavelength

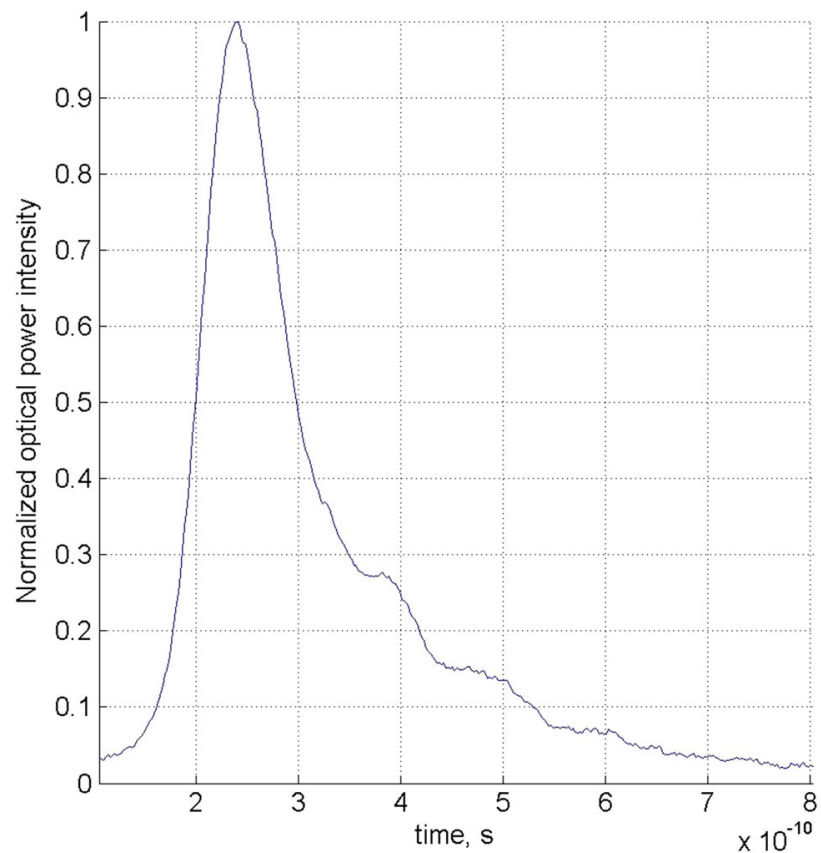

Fig. 9 Time domain integration of streak camera measurement result of the $30 \mu \mathrm{m} 3.0 \mathrm{~mm}$ quantum well laser diode

measurement of the time-domain shape of the optical pulse was expected with the streak camera measurement.

The triggering signal of the streak camera sweep unit was produced from the laser diode current pulse which has significantly smaller jitter with respect to the optical pulse than that measured from the driver input. Using this approach, a FWHM of the jitter between current pulse and streak camera sweep triggering pulse was $\sim 40$ ps.

A 3D plot of the streak camera measurement results is shown in Fig. 8.

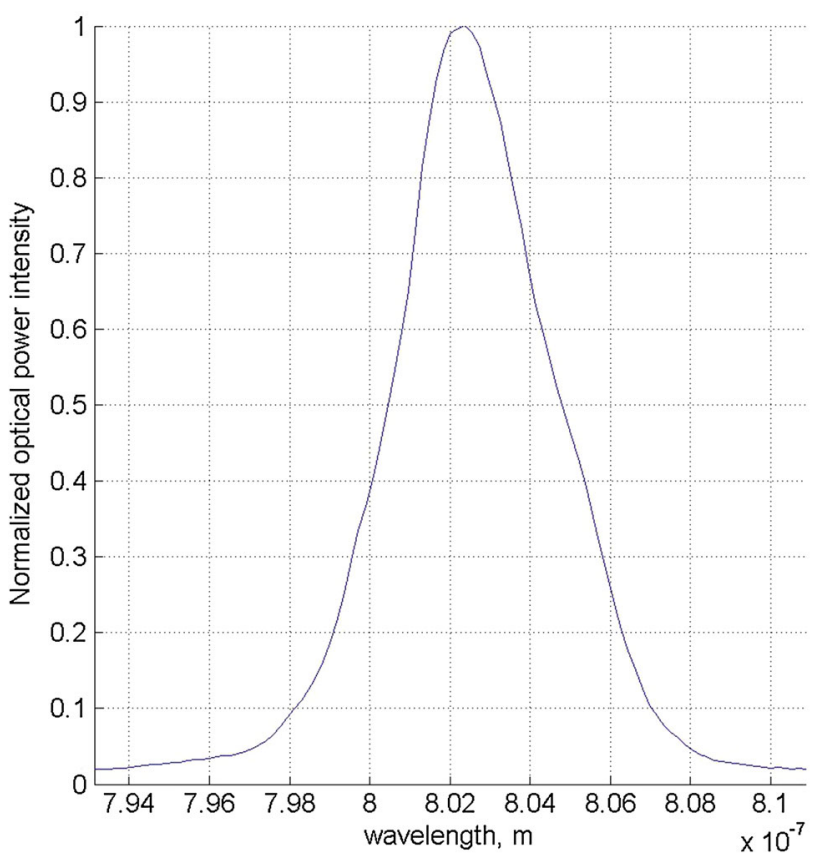

Fig. 10 Wavelength domain integration of streak camera measurement result of the $30 \mu \mathrm{m} 3.0 \mathrm{~mm}$ quantum well laser diode

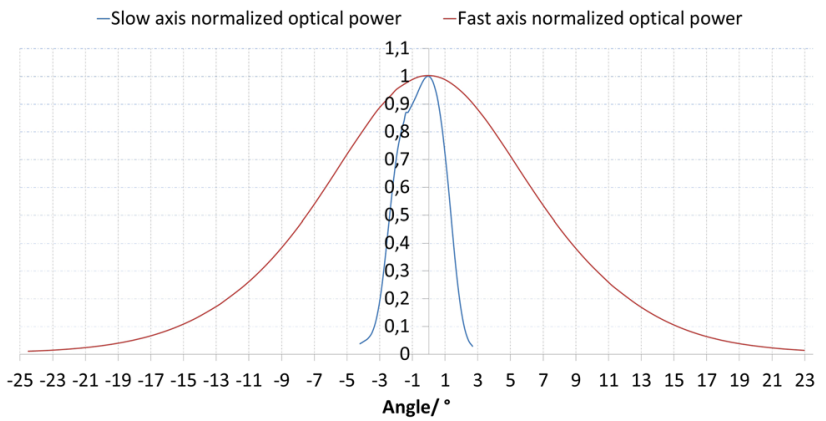

Fig. 11 Fast and slow axis beam divergence of the $30 \mu \mathrm{m} 3.0 \mathrm{~mm}$ quantum well laser diode

The time and wavelength domain curves given in Figs. 9 and 10 are normalized instantaneous optical pulse power and the spectrum of the average power integrated from the streak camera measurement result data shown in Fig. 8. The spectrum shows a center wavelength of approximately $802 \mathrm{~nm}$ and a FWHM of $4 \mathrm{~nm}$, as can be seen in Fig. 10.

A far field distribution measurement was performed to determine the QW laser diode's beam divergence and thus to estimate the size of the required transmitter optics. The laser diode transmitter was attached on a turn table with two perpendicular orientations and the emitted average power was measured with an average optical power meter, applying a $2 \mathrm{~mm}$ entrance slit, $1 \mathrm{~m}$ away from the laser diode. From the results given in Fig. 11, the fast and slow axis FWHM of $15^{\circ}$ and $4^{\circ}$, respectively, can be seen. From the practical point of view, the small wide axis value of $15^{\circ}$ 


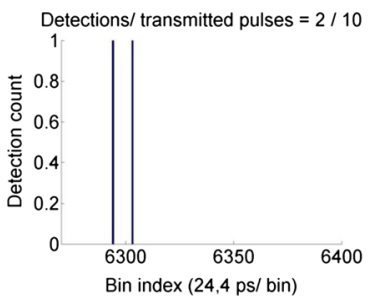

a

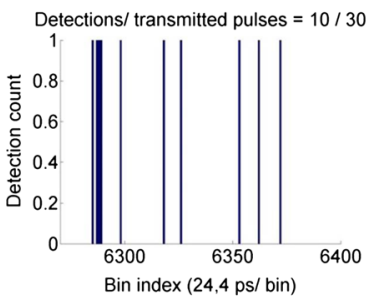

C

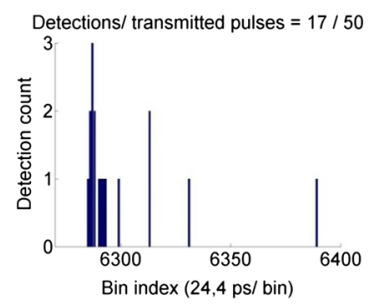

e

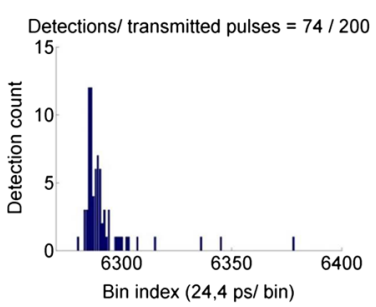

g

Detections $/$ transmitted pulses $=182 / 800$ 25

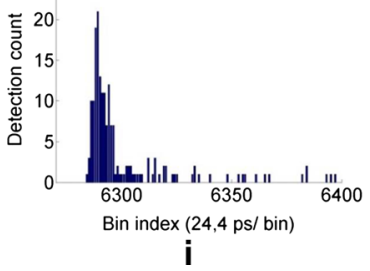

Fig. 12 Time of flight histograms with varying number of transmitted total pulse counts

is highly desirable since it keeps the size of the transmitter optics reasonable while providing a high optical energy collection efficiency.

\section{Laser radar experiments}

Some preliminary system level measurements were done to get an idea of overall performance of the single photon detection based laser radar. The transmitter consisted of the
$30 \mu \mathrm{m}$ wide, $3.0 \mathrm{~mm}$ long quantum well laser diode, and the receiver used a single $\sim 20 \mu \mathrm{m}$ CMOS SPAD element with a receiver aperture of $18 \mathrm{~mm}$. The system's performance parameters of interest were the achievable detection rate and the single shot precision. The target was a black rubber surface (reflectance $\sim 3 \%$ ) at a distance of $\sim 25 \mathrm{~m}$ from the radar. Transmitted laser pulse counts were 10, 20, 30, 40, 50, 100, 200, 400 and 800 and each pulse count measurement was repeated ten times. The applied pulsing frequency was $100 \mathrm{kHz}$. Measurements were performed in normal indoor lightning conditions.

Measurement results presented in Fig. 12 show TOF histograms of the above mentioned pulse counts. The hit counts in individual measurements were $2,8,10,17,17$, $34,74,101$ and 182, and thus the detection rate varies between 20 and $40 \%$. The cumulative shape of the results distribution resembles a convolution of the optical pulse and the SPAD time-domain response which is becoming increasingly pronounced with increasing pulse counts. Most of the hits are concentrated around the expected flight time, corresponding to the distance to the target, and show also the expected diffusion tail characteristic of CMOS SPADs [5]. The single shot precision based of the time-offlight (TOF) histograms is $\sim 20 \mathrm{~mm}$ (FWMH).

\section{Conclusions}

It is shown experimentally that high energy, short ( 100 ps) optical pulses can be produced with semiconductor lasers with a large equivalent spot size, or the ratio $\left(d_{a} / \Gamma_{a}\right)$ of the active layer thickness $d_{a}$ to the confinement factor $\Gamma_{a}$. QW and bulk laser diodes with $d_{a} / \Gamma_{a} \approx 3 \mu \mathrm{m}$ and stripe width/cavity length of $30 \mu \mathrm{m} / 3 \mathrm{~mm}$ were realized, and achieved a pulse energy of the order of $\sim 1 \mathrm{~nJ}$ and a pulse length of about 100 ps, when pumped by a current pulse with an amplitude of 6-8 A and a duration of 1 ns. The far-field characteristics were also studied with a view to establishing the time-homogeneity and energy distribution. The wide axis value of the far-field distribution was measured to be $15^{\circ}$ for a $\mathrm{QW}$ laser diode, which makes it possible to achieve high optical energy collection and low divergence with a low $\mathrm{F}$ number of the transmitter optics.

It was also demonstrated that an energy level of $\sim 1 \mathrm{~nJ}$ is quite sufficient for radar measurements for distances up to tens of meters to non-cooperative targets with a receiver aperture of $<20 \mathrm{~mm}$. For example, a black rubber target with a reflectance of $\sim 3 \%$ gave a detection rate of $20-40 \%$ at a distance of $25 \mathrm{~m}$. We conclude that bulk and quantum well laser diodes utilizing the "enhanced gain switching" principle based on the large equivalent spot size are quite promising candidates for the role of a transmitter 
in single photon detection based laser radar devices and applications, since they allow considerable miniaturization in the transmitter part of the laser radar.

Open Access This article is distributed under the terms of the Creative Commons Attribution 4.0 International License (http://crea tivecommons.org/licenses/by/4.0/), which permits unrestricted use, distribution, and reproduction in any medium, provided you give appropriate credit to the original author(s) and the source, provide a link to the Creative Commons license, and indicate if changes were made.

\section{References}

1. Niclass, C., Soga, M., Matsubara, H., Ogawa, M., Kagami, M.: A 0.18- $\mu \mathrm{m}$ CMOS SoC for a 100-m-Range 10-Frame/s $200 \times 96-$ pixel time-of-flight depth sensor. IEEE J. Solid State Circuits 49(1), 315-330 (2014)

2. Ito, K., Niclass, C., Aoyagi, I., Matsubara, H., Soga, M., Kato, S., Maeda, M., Kagami, M.: System design and performance characterization of a MEMS-based laser scanning time-of-flight sensor based on a $256 \times 64$-pixel single-photon imager. IEEE Photonics J. Vol. 5, Issue 2, 15p., 2013

3. Perenzoni, D., Gasparini, L., Massari, N., Stoppa, D.: Depthrange extension with folding technique for SPAD-based TOF LIDAR systems. Proceedings of the IEEE SENSORS Conference, pp. 622-624 (2014)

4. Kostamovaara, J., Huikari, J., Hallman, L., Nissinen, I., Nissinen, J., Rapakko, H., Avrutin, E., Ryvkin, B.: On laser ranging based on high-speed/energy laser diode pulses and single-photon detection techniques. IEEE Photonics J. 7(2), 1-15 (2015)

5. Pancheri, L., Stoppa, D.: Low-Noise CMOS single-photon avalanche diodes with $32 \mathrm{~ns}$ dead time. Proceedings of the 37th European Solid State Device Research Conference, ESSDERC, pp. 362-365 (2007)
6. Veerappan, C., Charbon, E.: CMOS SPAD Based on photo-carrier diffusion achieving PDP $>40 \%$ from 440 to $580 \mathrm{~nm}$ at $4 \mathrm{~V}$ excess bias. IEEE Photonics Technol. Lett. 27(23), 2445-2448 (2015)

7. Ryvkin, B.S., Avrutin, E.A., Kostamovaara, J.: Asymmetric waveguide laser diode for high-power optical pulse generation by gain switching. J. Lightw. Technol. 27(12), 2125-2131 (2009)

8. Ryvkin, B.S., Avrutin, E.A., Kostamovaara, J.: Quantum well laser with an extremely large active layer width to optical confinement factor ratio for high-energy single picosecond pulse generation by gain switching. Semicond. Semicond. Sci. Technol. 26(4), 045010 (2011). (pp. 1-4)

9. Lanz, B., Ryvkin, B.S., Avrutin, E.A., Kostamovaara, J.: Performance improvement by a saturable absorber in gain-switched asymmetric-waveguide laser diodes. Opt. Express 21(24), 29780-29791 (2013)

10. Avrutin, E.A., Ryvkin, B.S., Kostamovaara, J.T., Kuksenkov, D.V.: Strongly asymmetric waveguide laser diodes for high brightness picosecond optical pulses generation by gain switching at $\mathrm{GHz}$ repetition rates. Semicond. Sci. Technol. 30(5), 055006 (2015)

11. Huikari, J., Avrutin, E., Member, I.E.E.E., Ryvkin, B., Nissinen, J., Kostamovaara, J., Senior Member, IEEE: High-energy picosecond pulse generation by gain switching in asymmetric waveguide structure multiple quantum well lasers. IEEE J. Select. Topics Quantum Electron. 21(6), 1-6 (2015)

12. Hallman, L., Huikari, J., Kostamovaara, J.: A high-speed/power laser transmitter for single photon imaging applications. Proceedings of the IEEE SENSORS Conference, pp. 1157-1160 (2014)

13. Ryvkin, B.S., Avrutin, E.A., Kostamovaara, J.: Narrow versus broad asymmetric waveguides for single-mode high-power laser diode. J. Appl. Phys. 113, 013104 (2013)

14. Becker, W.: Advanced time-correlated single photon counting techniques. Springer, Germany (2005) 